\title{
Non-equilibrium Fluctuation Relations in a Quantum Coherent Conductor
}

\author{
Shuji Nakamura ${ }^{1 \dagger}$, Yoshiaki Yamauchi ${ }^{1 \dagger}$, Masayuki Hashisaka ${ }^{1}$, Kensaku Chida ${ }^{1}$, Kensuke Kobayashi米 and \\ Teruo Ono ${ }^{1}$ Renaud Leturcq ${ }^{2}$, Klaus Ensslin ${ }^{3}$, Keiji Saito ${ }^{4}$, Yasuhiro Utsumi ${ }^{5}$, Arthur C. Gossard ${ }^{6}$ \\ ${ }^{1}$ Institute for Chemical Research, Kyoto University, Uji, Kyoto 611-0011, Japan. \\ ${ }^{2}$ Institute of Electronics, Microelectronics and Nanotechnology, CNRS-UMR 8520, \\ Department ISEN, Avenue Poincaré 59652 Villeneuve d'Ascq, France. \\ ${ }^{3}$ Solid State Physics Laboratory, ETH Zürich, CH-8093 Zürich, Switzerland. \\ ${ }^{4}$ Graduate School of Science, University of Tokyo, Tokyo 113-0033, Japan. \\ ${ }^{5}$ Institute for Solid State Physics, University of Tokyo, Kashiwa, Chiba 277-8581, Japan. \\ ${ }^{6}$ Materials Department, University of California, Santa Barbara, California 93106, USA, and \\ ${ }^{\dagger}$ these authors equally contributed to this work.
}

(Dated: November 3, 2018)

\begin{abstract}
We experimentally demonstrate the validity of non-equilibrium fluctuation relations by using a quantum coherent conductor. In equilibrium the fluctuation-dissipation relation leads to the correlation between current and current noise at the conductor, namely, Johnson-Nyquist relation. When the conductor is voltage-biased so that the non-linear regime is entered, the fluctuation theorem has predicted similar non-equilibrium fluctuation relations, which hold true even when the Onsager-Casmir relations are broken in magnetic fields. Our experiments qualitatively validate the predictions as the first evidence of this theorem in the non-equilibrium quantum regime.
\end{abstract}

PACS numbers: 05.40.-a, 72.70.+m, 73.23.-b, 85.35.Ds

The fluctuation-dissipation relation [1] is a central concept in physics. It was triggered by the description of Brownian motion by Einstein [2], who claimed that the response of a physical system to an external force is proportional to its equilibrium fluctuation. In electrical circuits this fact manifests itself as the Johnson-Nyquist (JN) relation [3, 4] in such a way that the conduction through a conductor is proportional to its current noise in equilibrium. The linear response theory [5, 6] founded on this relation together with the Onsager-Casimir reciprocal relations [7] provides a powerful tool to describe a variety of physical systems. This is, however, justified only when the systems are close to equilibrium, and the generalization to the non-equilibrium regime has been long sought.

Generally the current $(I)$ passing through a conductor can be expressed as a polynomial of the bias voltage $(V)$ as follows;

$$
I=G_{1} V+\frac{1}{2 !} G_{2} V^{2}+\frac{1}{3 !} G_{3} V^{3}+\cdots,
$$

where the first term represents Ohm's law with conductance $G_{1}$ (Such an expansion in $V$ is assumed to be valid for mesoscopic transport [8]). While in a mesoscopic conductor $G_{1}$ is directly related to the transmission [9] the higher-order coefficients $\left(G_{2}, G_{3}, \ldots\right)$ convey information on electron-electron interactions in a voltage-biased conductor as shown recently [10 13]. Similarly to Eq. (1), a polynomial of $V$ for the current noise power $S$ generated in the conductor, namely the variance of $I$, can be expressed as;

$$
S=S_{0}+S_{1} V+\frac{1}{2 !} S_{2} V^{2}+\cdots,
$$

The coefficients of the first terms in Eqs. (1) and (2) are linked by the JN relation $S_{0}=4 k_{B} T G_{1}$, where $k_{B}$ is the Boltzmann constant and $T$ is the temperature of the conductor [3, 4, 14]. Now, an essential question arises: Are there also correlations between the higher orders (in voltage) in current $I$ and noise power $S$ ?

Here we demonstrate a non-equilibrium fluctuation relation in a quantum coherent regime. We find a proportionality between $S_{1}$ and $G_{2}$, which corresponds to the next order correlation beyond the JN relation between $S_{0}$ and $G_{1}$. The present relation is asymmetric in magnetic fields unlike the Onsager-Casimir symmetry and is valid even in the presence of interactions. Such a direct link between non-linearity in voltage and fluctuations out-ofequilibrium has been predicted theoretically [15 19$]$ by applying the fluctuation theorem [20] to a quantum coherent conductor. Our experiments qualitatively validate the predictions but disagree on the quantitative level.

We used an Aharonov-Bohm (AB) ring as a typical coherent conductor. Figure 1(a) shows an atomic force microscope (AFM) image of the AB ring fabricated by local oxidation using an AFM 21] on a GaAs/AlGaAs heterostructure two-dimensional electron gas (2DEG) (the electron density $3.7 \times 10^{11} \mathrm{~cm}^{-2}$, the mobility $2.7 \times 10^{5}$ $\mathrm{cm}^{2} / \mathrm{Vs}$, and the electron mean free path $2.7 \mu \mathrm{m}$ at zero back-gate voltage) as well as the experimental setup for the two-terminal measurement in a dilution refrigerator. As our 2DEG has a back gate to tune the electron density, the conductance of the $\mathrm{AB}$ ring can be modulated by the back gate voltage $V_{g}$ and the magnetic field $B$ (the $\mathrm{AB}$ effect). Figure 1(b) shows the conductance as a function of $V_{g}$ and $B$, displaying clear $\mathrm{AB}$ oscillations with an oscillation period being $25 \mathrm{mT}$, in agreement with the ring radius of $230 \mathrm{~nm}$ [13]. The conductance 

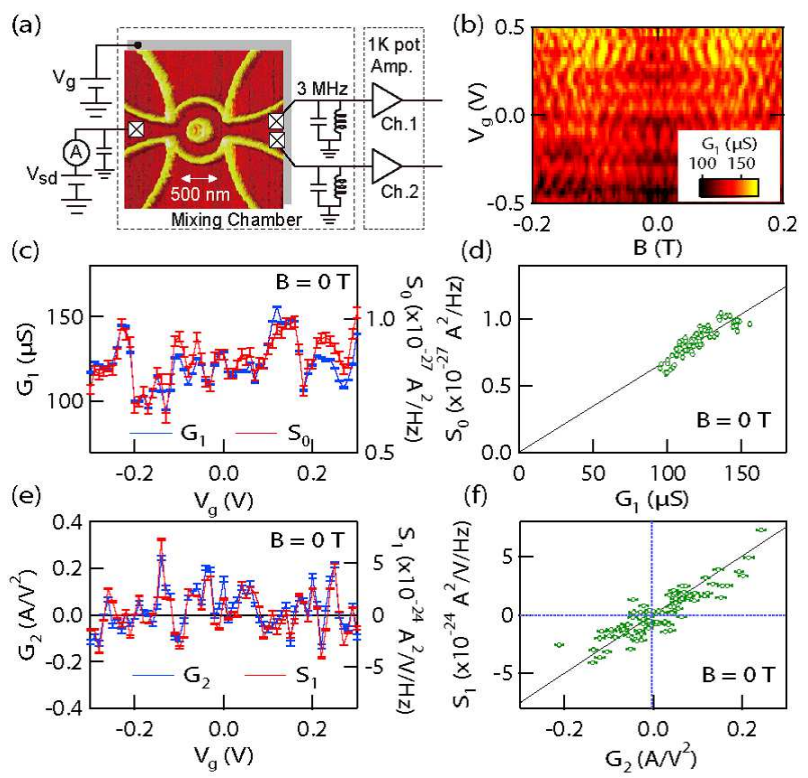

(d)
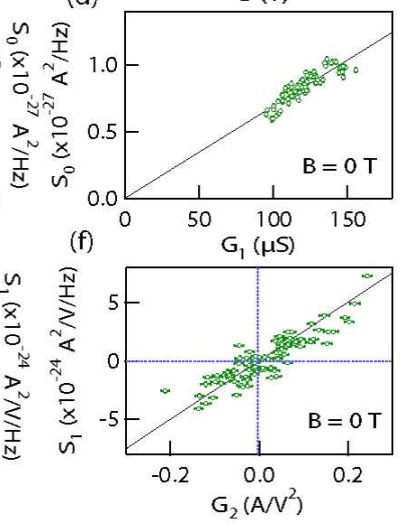

FIG. 1. (a) AFM image of the AB ring with the DC and noise measurement setup in the dilution refrigerator whose base temperature is $45 \mathrm{mK}$. The in-plane gates defined by the oxide lines are grounded in this experiment. (b) Conductance of the $\mathrm{AB}$ ring as a function of $V_{g}$ and $B$. (c) $G_{1}$ (left axis) and $S_{0}$ (right axis) as a function of $V_{g}$ at $B=0$ T. (d) $S_{0}$ is plotted as a function of $G_{1}$. The solid line indicates the JN relation of $S_{0}=4 k_{B} T G_{1}$ with $T=125 \mathrm{mK}$. (e) $G_{2}$ (left axis) and $S_{1}$ (right axis) as a function of $V_{g}$. (f) $S_{1}$ is plotted as a function of $G_{2}$. The solid line is the result of the fitting $\left(S_{1}=3.64 \times 4 k_{B} T G_{2}\right.$ with $\left.T=125 \mathrm{mK}\right)$.

of the ring at $B=0 \mathrm{~T}$ and $V_{g}=0 \mathrm{~V}$ is 3.1 in units of $e^{2} / h \sim(25.8 \mathrm{k} \Omega)^{-1}$. The visibility of the $\mathrm{AB}$ oscillations around $B=0 \mathrm{~T}$ is 0.13 (see Fig. 3(a)).

In addition to the DC measurement, we performed a noise measurement as follows (see Fig. 1(a)). The voltage fluctuation across the sample on the resonant circuit, whose resonance frequency is about $3.0 \mathrm{MHz}$ with the bandwidth of $\sim 140 \mathrm{kHz}$, is extracted as an output signal of the cryogenic amplifier 22 24]. The time-domain signal is then captured by a two-channel digitizer, and is converted to spectral density data via FFT, where the cross-correlation technique is adopted to increase the resolution. By numerically fitting the obtained resonance peak, the current noise power spectral density $S$ is deduced as performed in Ref. [23].

We deduce the coefficients in Eqs. (1) and (2) by numerically fitting the measured current $I$ and current noise power spectral density $S$ as polynomials of $V$ [25]. In the analysis we set the bias window to $|e V| \leq 50 \mu \mathrm{eV}$, where Joule heating is negligible as confirmed in previous noise measurements 24] (Two examples of the analysis are shown in Fig. 2). Figure 1(c) shows the conductance $G_{1}$ (left axis) and the equilibrium noise power density $S_{0}$ (right axis) as a function of $V_{g}$ at $B=0 \mathrm{~T}$. Due to electron interferences, $G_{1}$ varies as $V_{g}$ changes. The behavior

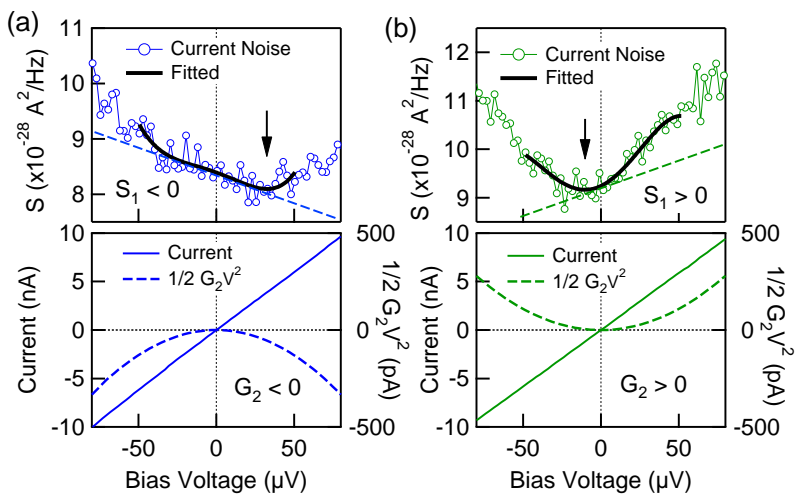

FIG. 2. (a) (b) (Lower panels) Two examples of $I-V$ characteristics obtained at different $V_{g}$ 's, where $\frac{1}{2} G_{2} V^{2}$ components deduced from the numerical fitting are superposed as dashed curves. (Upper panels) The corresponding $S$, where the results of the polynomial fitting and $S_{1} V$ components are superposed as solid and dashed curves, respectively. The nonequilibrium noises are smaller than the equilibrium ones at certain bias voltages indicated by the arrows. The signs of $G_{2}$ and $S_{1}$ are correlated (negative in (a) and positive in (b)).

of $S_{0}$ perfectly follows that of $G_{1}$ as expected from the JN relation. The proportionality between $G_{1}$ and $S_{0}$ shown in Fig. 1(d) indicates that $S_{0}=4 k_{B} T G_{1}$ is fulfilled with an electron temperature of $T=125 \mathrm{mK}$.

Now let us focus on the next order in voltage $V$. Figure 1(e) shows $G_{2}$ (left axis) and $S_{1}$ (right axis) as a function of $V_{g}$. Both $G_{2}$ and $S_{1}$ vary reflecting electron interferences. Moreover, their variations are correlated (Fig. 1(f)) with a correlation factor being 0.88 between the two. When the result is expressed in the form $S_{1}=4 k_{B} T G_{2} \alpha^{0}$ ("0" denotes the zero-field) based on the assumption that the functional form is similar to the JN relation, we obtain $\alpha^{0}=3.64 \pm 0.54$ by a numerical analysis [26], where the error bar indicates the 95\% confidence interval (see the solid line in Fig. 1(f)). While the magnitude of $\alpha^{0}$ will be discussed later, two remarks are made. First, a proportionality between $G_{2}$ and $S_{1}$ is already contained in the scattering theory for non-interacting systems [8], which yields general results to express the current and its noise as a function of the bias voltage, the transmission of the conductor, and the Fermi distribution of the reservoirs at a given temperature. However, we will show later that the present relation is valid beyond the description based on this picture in terms of the breaking of the Onsager-Casimir symmetry. Second, the finite values of $S_{1}$ indicate that there is a certain bias voltage range where we measure "negative excess noise", namely the non-equilibrium noise smaller than the equilibrium one [8, 18, 27]. Indeed, depending on the coefficients $\left(S_{1}, S_{2}, \ldots\right)$ in Eq. (2), the value of the bias voltage to give the minimum noise power is shifted from zero by a value of the order of $10 \mu \mathrm{V}$ [18] as shown in Fig. 2. 
The Onsager-Casimir reciprocity is a fundamental relation to characterize the response of a system close to equilibrium [7]. Figure 3(a) shows $G_{1}$ (left axis) and $S_{0}$ (right axis) as a function of $B$ at $V_{g}=0.02 \mathrm{~V}$. Both of them, displaying a periodic oscillations due to the $\mathrm{AB}$ effect, are symmetric with regard to $B=0 \mathrm{~T}$, namely $G_{1}(B)=G_{1}(-B)$ and $S_{0}(B)=S_{0}(-B)$. Furthermore, they are proportional to each other (Fig. 3(b)). This is the direct consequence of the $\mathrm{JN}$ relation and the Onsager-Casimir reciprocity. On the other hand, the next order coefficient $G_{2}$ has no such symmetry as shown in Figs. 3(c) and 3(e) (blue curves), where the symmetric components $G_{2}^{S}(B) \equiv G_{2}(B)+G_{2}(-B)$ and antisymmetric components $G_{2}^{A}(B) \equiv G_{2}(B)-G_{2}(-B)$ are presented, respectively. The emergence of a finite $G_{2}^{A}$ at $B \neq 0$ is in agreement with previous works on the breaking of the Onsager-Casimir reciprocity in the non-linear transport regime 10 13. It was attributed to electronelectron interactions in mesoscopic conductors 10, 11] since, in the Landauer picture for non-interacting electrons [8], the transmission probability of electrons always obeys the Onsager-Casimir reciprocity regardless of applied bias voltages, leading to a vanishing anti-symmetric term $G_{2}^{A}$.

Here we show that the non-equilibrium relation between $G_{2}$ and $S_{1}$ is valid even when the Onsager-Casimir relation is broken. In Figs. 3(c) and 3(e) the symmetric components $S_{1}^{S}(B) \equiv S_{1}(B)+S_{1}(-B)$ and antisymmetric components $S_{1}^{A}(B) \equiv S_{1}(B)-S_{1}(-B)$ as a function of $B$ are superposed in red curves, respectively. In addition to the proportionality between $G_{2}^{S}$ and $S_{1}^{S}$ (see Fig. 3(d)), there exists a clear proportionality between $G_{2}^{A}$ and $S_{1}^{A}$ as shown in Fig. 3(f). The correlation factors are 0.94 and 0.88 for the symmetric and anti-symmetric parts, respectively. For the symmetric part, $\alpha^{S}=2.69_{-0.35}^{+0.59}$ is obtained in the expression of $S_{1}^{S}=4 k_{B} T G_{2}^{S} \alpha^{S}$, while $\alpha^{A}=2.18_{-0.18}^{+0.32}$ is deduced in the anti-symmetric relation $S_{1}^{A}=4 k_{B} T G_{2}^{A} \alpha^{A}$. Importantly, the anti-symmetric part is non-trivial in that it is the consequence of the departure of the system from the Onsager-Casimir symmetry, and a signature of nonequilibrium and non-linearity. Nevertheless, the higherorder correlation still exists there as well as in the symmetric part, but with different coefficients.

What is the reason for the correlations in the nonlinear transport regime? Here we give an intuitive picture of higher order correlations following recent theories [15-19]. Electron transport can be viewed as the electron exchange process between two reservoirs via a conductor. Consider the probability $P(Q)$ that one reservoir gains $Q$ electrons from the other as a consequence of the exchange (in the absence of a magnetic field, for simplicity). As time reversal symmetry, particle-number conservation, and energy conservation are required over the whole system including the reservoirs, $P(Q)$ should satisfy $P(Q)=P(-Q) \exp \left(\frac{e V}{k_{B} T} Q\right)[15-19]$. This means
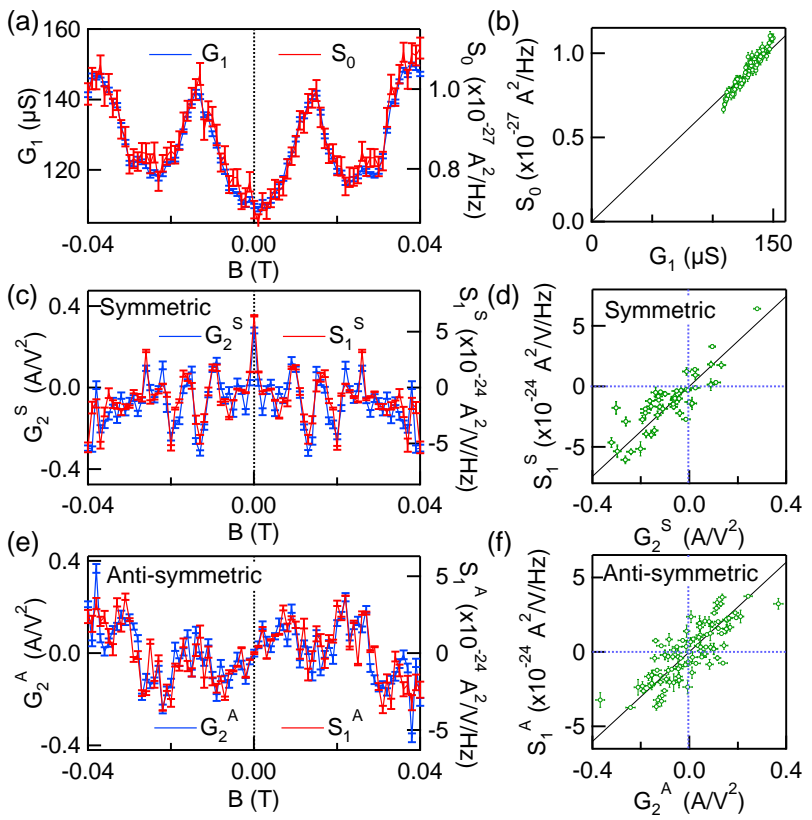

FIG. 3. (a) $G_{1}$ (left axis) and $S_{0}$ (right axis) as a function of $B$ at $V_{g}=0.02 \mathrm{~V}$. (b) $S_{0}$ as a function of $G_{1}$. The solid line $S_{0}=4 k_{B} T G_{1}$ is the JN relation at $T=125 \mathrm{mK}$. (c) Symmetric components in the second-order coefficients $G_{2}^{S}$ (left axis) and $S_{1}^{S}$ (right axis) as a function of $B$. (d) $S_{1}^{S}$ as a function of $G_{2}^{S}$. The solid line is the result of the fitting corresponding to $S_{1}^{S}=2.69 \times 4 k_{B} T G_{2}^{S}$ for $T=125 \mathrm{mK}$. (e) Anti-symmetric components in the second-order coefficients $G_{2}^{A}$ (left axis) and $S_{1}^{A}$ (right axis) as a function of $B$. (f) $S_{1}^{A}$ as a function of $G_{2}^{A}$. The solid line is the result of the fitting corresponding to $S_{1}^{A}=2.18 \times 4 k_{B} T G_{2}^{A}$ for $T=125 \mathrm{mK}$.

that the probability of a $Q$-electrons exchange process and that of its time-reversed process ( $-Q$-electrons exchange) are linked with a factor difference of $\exp \left(\frac{e V}{k_{B} T} Q\right)$, reflecting a probability difference of the initial states for the two exchange processes. Note that this equality based on the micro-reversibility is valid even in the non-equilibrium situation and provides the basis of the fluctuation theorem [20]. As $P(Q)$ contains all the information associated with electron transport through the conductor, the strong constraint posed by this equality on the cumulants of $Q$ yields a number of correlations starting with the JN relation. For the second order, the equality predicts $S_{1}=2 k_{B} T G_{2}[15-19]$. In the presence of magnetic fields, the fluctuation relations that do not rely on the microreversibility out of equilibrium were recently pointed out [17, 18], where the anti-symmetric relation is expressed by $S_{1}^{A}-2 k_{B} T G_{2}^{A}=C_{0}^{A} / 3 k_{B} T$. Here, $C_{0}^{A}$ is the anti-symmetric part of the third cumulant ("skewness") at equilibrium. With the restriction of micro-reversibility, this anti-symmetric relation [16] is reduced to $S_{1}^{A}=6 k_{B} T G_{2}^{A}$ with another relation $S_{1}^{A}=C_{0}^{A} / 2 k_{B} T$. In both cases, the symmetric relation is given by $S_{1}^{S}=2 k_{B} T G_{2}^{S}$. 


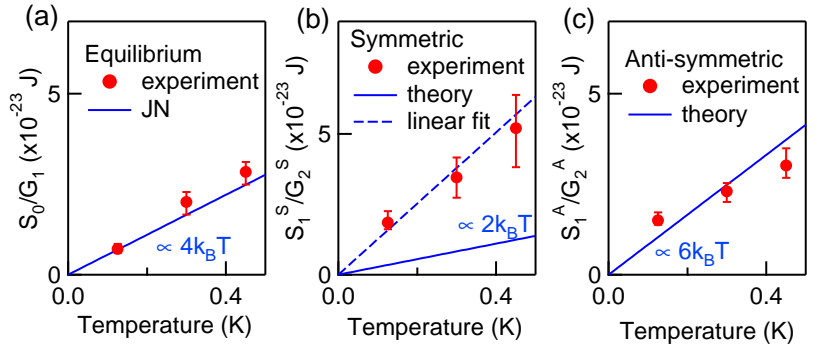

FIG. 4. (a) Values of $S_{0} / G_{1}$ obtained at 125,300 , and $450 \mathrm{mK}$. The solid line shows the JN relation. (b) Values of $S_{1}^{S} / G_{2}^{S}$ at 125,300 , and $450 \mathrm{mK}$. The solid line is the theoretical expectation $\left(S_{1}^{S} / G_{2}^{S}=2 k_{B} T\right)[15-19]$. The dashed line is the result of the linear fitting that gives $S_{1}^{S} / G_{2}^{S}=4.6 \times 2 k_{B} T$. (c) Values of $S_{1}^{A} / G_{2}^{A}$ at 125,300 , and $450 \mathrm{mK}$. The solid line is the theoretical expectation $\left(S_{1}^{A} / G_{2}^{A}=6 k_{B} T\right)$ [16, 19].

At zero-field, the observed linearity between $S_{1}$ and $G_{2}$ qualitatively agrees with the above discussion, although the theory does not well reproduce the obtained ratio $S_{1} / 2 k_{B} T G_{2}=2 \alpha^{0} \sim 7$. At finite magnetic field as well, while the expected proportionality is obtained, the ratio $\left(S_{1}^{S} / 2 k_{B} T G_{2}^{S}=2 \alpha^{S} \sim 5\right)$ is larger than in theory. The present observation for $\alpha^{0}$ and $\alpha^{S}$ is perfectly reproducible in our experiments performed in several different conditions for different magnetic fields and gate voltages. Concerning the temperature dependence, although the ratio $S_{0}^{S} / G_{1}^{S}$ follows the JN relation (Fig. 4(a)), the ratio $S_{1}^{S} / G_{2}^{S}$ shown in Fig. 4(b) depends linearly on temperature as expected, but the slope is similarly larger than the theoretical prediction. This implies the necessity of further theoretical work that takes realistic situations into account 28. In contrast, the result for the anti-symmetric component $\left(S_{1}^{A} / 6 k_{B} T G_{2}^{A} \sim 1.5\right)$ is in better agreement with theory $S_{1}^{A}=6 k_{B} T G_{2}^{A}$ in spite of a possible independent contribution of $C_{0}^{A}$ when only the universal relation without micro-reversibility $\left(S_{1}^{A}-2 k_{B} T G_{2}^{A}=C_{0}^{A} / 3 k_{B} T\right)$ is assumed [17, 18]. The agreement is also clear in the temperature dependence of the ratio $S_{1}^{A} / G_{2}^{A}$ as shown in Fig. 4(c). This observation suggests that micro-reversibility in magnetic field is likely to be validated in the present experiment.

In conclusion we have experimentally proven the presence of the direct link between the non-linear response and the non-equilibrium fluctuation in the $\mathrm{AB}$ ring, as theoretically predicted on the basis of the fluctuation theorem. While our demonstration was performed for the simplest case in a normal coherent conductor without energy relaxation in the scattering region, the present finding will further open an applicability of the fluctuation theorem in the quantum coherent regime and in the presence of magnetic fields.

We appreciate fruitful discussions from Markus Büttiker, Masahito Ueda and Takeo Kato. This work is partially supported by KAKENHI, Yamada Science
Foundation, SCAT, Matsuo Science Foundation, Strategic International Cooperative Program the Japan Science and Technology Agency (JST), and the German Science Foundation (DFG).

* kensuke@scl.kyoto-u.ac.jp

[1] H. B. Callen, and T. A. Welton, Phys. Rev. 83, 34 (1951).

[2] A. Einstein, Ann. Phys. 17, 549 (1905).

[3] J. B. Johnson, Phys. Rev. 32, 97 (1928).

[4] H. Nyquist, Phys. Rev. 32, 110 (1928).

[5] M. S. Green, J. Chem. Phys. 22, 398 (1954).

[6] R. Kubo, J. Phys. Soc. Jpn. 12, 570 (1957).

[7] L. Onsager, Phys. Rev. 38, 2265 (1931); H. B. G. Casimir, Rev. Mod. Phys. 17, 343 (1945).

[8] Y. M. Blanter, and M. Büttiker, Phys. Rep. 336, 1 (2000).

[9] R. Landauer, IBM J. Res. Dev. 1, 223 (1957).

[10] D. Sanchéz, and M. Büttiker, Phys. Rev. Lett. 93, 106802 (2004); M. L. Polianski, and M. Büttiker, Phys. Rev. B 76, 205308 (2007).

[11] B. Spivak, and A. Zyuzin, Phys. Rev. Lett. 93, 226801 (2004).

[12] J. Wei et al., Phys. Rev. Lett. 95, 256601 (2005); D. M. Zumbühl, C. M. Marcus, M. P. Hanson, and A.C. Gossard, ibid. 96, 206802 (2006); C. A. Marlow et al., ibid. 96, 116801 (2006); L. Angers et al., Phys. Rev. B 75, 115309 (2007).

[13] R. Leturcq et al., Phys. Rev. Lett. 96, 126801 (2006); R. Leturcq et al., Physica E 35, 327-331 (2006).

[14] The conventional shot noise formula based on the scattering approach [8] can be also expressed as Eq. (2).

[15] J. Tobiska, and Yu. V. Nazarov, Phys. Rev. B 72, 235328 (2005).

[16] K. Saito, and Y. Utsumi, Phys. Rev. B 78, 115429 (2008); Y. Utsumi and K. Saito, ibid. 79235311 (2009).

[17] H. Förster, and M. Büttiker, Phys. Rev. Lett. 101, 136805 (2008).

[18] H. Förster, and M. Büttiker, arXiv:0903.1431

[19] D. Andrieux, P. Gaspard, T. Monnai, and S. Tasaki, New J. Phys. 11, 043014 (2009).

[20] D. J. Evans, E. G. D. Cohen, and G. P. Morriss, Phys. Rev. Lett. 71, 2401 (1993).

[21] R. Held et al., Appl. Phys. Lett. 73, 262 (1998).

[22] R. de-Picciotto et al., Nature 389, 162 (1997); M. Reznikov et al., ibid. 399, 238 (1999).

[23] L. DiCarlo et al., Rev. Sci. Instrum. 77, 073906 (2006); M. Hashisaka et al., J. Phys. Conf. Series 109, 012013 (2008).

[24] M. Hashisaka et al., Phys. Rev. B 78, 241303(R) (2008); S. Nakamura et al., ibid. 79, 201308(R) (2009).

[25] The polynomial fitting was performed by taking up to the fifth order of $V$ for $I$ and up to the fourth order of $V$ for $S$ into account, respectively. The analysis up to third or seventh order of $V$ for $I$ and second order of $V$ for $S$ yields results consistent with those presented here.

[26] H. Passing, and W. A. Bablok, J. Clin. Chem. Clin. Biochem. 21, 709 (1983); As both $S_{1}\left(S_{1}^{S}\right.$ or $\left.S_{1}^{A}\right)$ and $G_{2}\left(G_{2}^{S}\right.$ or $\left.G_{2}^{A}\right)$ have statistical uncertainties, PassingBablok regression is more appropriate than the conventional least-square linear fitting to statistically estimate 
the error bars of the coefficients between the two values. [27] G. B. Lesovik, and R. Loosen, Z. Phys. B 91, 531 (1993).

[28] Quantitative disagreement between the experiment in the classical regime and the theory derived from the fluctuation theorem is discussed recently [Y. Utsumi et al., arXiv:0908.0229.

\section{Appendix: Simple deduction of the higher order correlations}

We explain the fluctuation theorem using the simplest setup. We consider a mesoscopic conductor, say a quantum point contact, where the two quantum wires are coupled by tunneling. For simplicity, no magnetic field is applied. The present system is described by the following Hamiltonian

$$
H=H_{L}+H_{R}+H_{L R},
$$

where $H_{L}$ and $H_{R}$ are the Hamiltonian of the left and right quantum wires and $H_{L R}$ is the tunneling part between them. The initial density matrix is decoupled into the equilibrium states of each wire, where the left and right wires are assumed to have equal temperature $1 / \beta$ and have chemical potentials $\mu_{L}$ and $\mu_{R}$, respectively. Then the whole density matrix is described by

$$
\begin{aligned}
\hat{\rho}_{\text {initial }} & =\sum_{n_{L}, n_{R}} \rho_{n_{L}, n_{R}}\left|n_{L}, n_{R}\right\rangle\left\langle n_{L}, n_{R}\right|, \\
\rho_{n_{L}, n_{R}} & =\frac{e^{-\beta\left[E_{n_{L}}-\mu_{L} n_{L}\right]}}{Z_{L}} \frac{e^{-\beta\left[E_{n_{R}}-\mu_{R} n_{R}\right]}}{Z_{R}}
\end{aligned}
$$

where $Z_{L}$ and $Z_{R}$ are the normalization factors, and $\left|n_{L}, n_{R}\right\rangle$ defines the state that $n_{L}$ and $n_{R}$ electrons are present inside the left and right wires with the eigenenergies $E_{n_{L}}$ and $E_{n_{R}}$ of $H_{L}$ and $H_{R}$, respectively. The probability to find the state $\left|n_{L}^{\prime}, n_{R}^{\prime}\right\rangle$ after a certain time $\tau$ starting from the initial state $\left|n_{L}, n_{R}\right\rangle$ is expressed as

$$
P_{\left(n_{L}, n_{R}\right) \rightarrow\left(n_{L}^{\prime}, n_{R}^{\prime}\right)}=\left|\left\langle n_{L}^{\prime}, n_{R}^{\prime}\left|e^{\frac{-i \tau}{\hbar} H}\right| n_{L}, n_{R}\right\rangle\right|^{2} \rho_{n_{L}, n_{R}} .
$$

We note the time reversal symmetry

$$
\left|\left\langle n_{L}^{\prime}, n_{R}^{\prime}\left|e^{\frac{-i \tau}{\hbar} H}\right| n_{L}, n_{R}\right\rangle\right|^{2}=\left|\left\langle n_{L}, n_{R}\left|e^{\frac{-i \tau}{\hbar} H}\right| n_{L}^{\prime}, n_{R}^{\prime}\right\rangle\right|^{2} .
$$

We also note electron number conservation $n_{L}-n_{L}^{\prime}=$ $-\left(n_{R}-n_{R}^{\prime}\right)$ and energy conservation satisfied at very large $\tau: E_{n_{L}^{\prime}}-E_{n_{L}} \approx-\left(E_{n_{R}^{\prime}}-E_{n_{R}}\right)$. Using these time reversal symmetry and conservation laws, we find the relation

$$
P_{\left(n_{L}, n_{R}\right) \rightarrow\left(n_{L}^{\prime}, n_{R}^{\prime}\right)}=P_{\left(n_{L}^{\prime}, n_{R}^{\prime}\right) \rightarrow\left(n_{L}, n_{R}\right)} e^{A\left(n_{L}-n_{L}^{\prime}\right)},
$$

where $A$ is an affinity $A=\beta\left(\mu_{L}-\mu_{R}\right)$. The probability that transmitted electron number is $Q$, is defined as $P(Q)=\sum_{n_{L}, n_{R}, n_{L}^{\prime}, n_{R}^{\prime}} P_{\left(n_{L}, n_{R}\right) \rightarrow\left(n_{L}^{\prime}, n_{R}^{\prime}\right)} \delta\left(Q-\left(n_{L}-n_{L}^{\prime}\right)\right)$. A direct consequence from the relation (A.6) is "Fluctuation Theorem":

$$
P(Q)=P(-Q) e^{A Q}
$$

Now let us discuss the higher order correlations between the current and its noise power, which are addressed in the present paper. With Fluctuation Theorem (A.6), we find the following identity

$$
\begin{aligned}
\langle Q\rangle & =\int d Q Q P(Q)=-\int d Q Q P(Q) e^{-A Q} \\
& =-\langle Q\rangle+A\left\langle Q^{2}\right\rangle-\frac{A^{2}}{2 !}\left\langle Q^{3}\right\rangle+\cdots
\end{aligned}
$$

Furthermore, we note that $\left\langle Q^{n}\right\rangle$ is a function of $A$, i.e.,

$$
\left\langle Q^{n}\right\rangle=\left\langle Q^{n}\right\rangle_{0}+A\left\langle Q^{n}\right\rangle_{1}+\frac{A^{2}}{2 !}\left\langle Q^{n}\right\rangle_{2}+\cdots
$$

Comparing order by order with respect to $A$, we find infinite number of relationships among these quantities, some of which are given as

$$
\begin{aligned}
& \left\langle Q^{2}\right\rangle_{0}=2\langle Q\rangle_{1} \\
& \left\langle Q^{2}\right\rangle_{1}=\langle Q\rangle_{2}
\end{aligned}
$$

Average current $I$ and current noise power $S$ are defined as $I=\langle Q\rangle / \tau$ and $S=2\left(\left\langle Q^{2}\right\rangle-\langle Q\rangle^{2}\right) / \tau$. The first relation A.9 is equivalent to the fluctuation dissipation $S_{0}=4 k_{\mathrm{B}} T G_{1}[\mathrm{G}$. Gallavotti, Phys. Rev. Lett. 77, 4334 - 4337 (1996)], and the second relation (A.10) is to $S_{1}=2 k_{\mathrm{B}} T G_{2}$ (See main text for the definitions of $G_{1}$, $G_{2}, S_{0}$, and $\left.S_{1}\right)$. More systematic and exact derivation for these relations including the finite magnetic field case is performed by using a cumulant generating function $[\mathrm{K}$. Saito \& Y. Utsumi, Phys. Rev. B 78, 115429 (2008)]. 